\title{
Ocular and Oral Complications
}

\author{
Shahrukh K. Hashmi
}

\subsection{Ocular Complications}

\subsubsection{Introduction}

Ocular complications in HSCT patients include both ocular GVHD- and non-GVHD-related late effects which, if left untreated, can lead to blindness. Non-GVHD associated complications include glaucoma, infections particularly (viral retinitis, fungal endophthalmitis), posterior segment abnormalities, and cataracts. Ocular GVHD is considered an umbrella term for both conjunctival disease and keratoconjunctivitis sicca, both of which can coexist simultaneously.

The World Health Organization considers blindness as one of the top causes for disability-adjusted life years, and it significantly affects the QoL. Thus, prevention and prompt management of ocular complications should be a priority of a transplant program given the morbidity associated with it.

\subsubsection{Ocular GVHD}

Though it is considered to be systematic disease, the pattern of isolated ocular GVHD manifesting

S. K. Hashmi $(\bowtie)$

Department of Adult Hematology, King Faisal

Specialist Hospital and Research Center,

Riyadh, Saudi Arabia

Mayo Clinic, Rochester, MN, USA

e-mail: shashmi@kfshrc.edu.sa later as GVHD in other organs is erratic (Townley et al. 2011). The 2014 NIH consensus development project on chronic GVHD does not account for therapeutic responses based mainly on patient-reported outcomes (PRO); thus the development of PRO measures (PROM) specific for ocular GVHD should be a priority of the GVHD research community. The NIH chronic GVHD consortium does recommend photoprotection, surveillance for infection, cataract formation, and increased intraocular pressure for prevention of ocular complications in GVHD - these measures should be routinely incorporated in the standard clinical practice of HSCT patients and are best achieved within the context of a multidisciplinary survivorship clinic along with ophthalmologists as part of the team.

The occurrence of ocular GVHD is variable in different series but is approximately 40 to $60 \%$ of patients receiving HSCT (Nassar et al. 2013). The risk factors for ocular GVHD include donorrecipient HLA and gender disparity (female donor to male recipient) and an older donor age.

The principles of ocular GVHD management include lubrication, drainage control, evaporation control, and minimization of ocular surface inflammation. The 2014 NIH chronic GVHD consensus panel has excellent recommendations for stepwise treatment of ocular GVHD which include preservative-free artificial tears for mild, topical cyclosporine or steroid eye drops for moderate/severe and oral pilocarpine or cevimeline for severe/moderate ocular GVHD, respectively (Carpenter et al. 
2015). Among surgical procedures for moderate/ severe ocular GVHD, punctal occlusion, superficial debridement of filamentary keratitis, and partial tarsorrhaphy have been recommended as surgeries with moderate-quality data on effectiveness. There is also some evidence on other modalities of treatment which include occlusive eye wear, lid care/warm compress/humidified environment, bandage contact lens, and gas-permeable scleral lenses. What is not clear is the optimal management algorithm for ocular GVHD with respect to medical versus surgical therapies and, within the surgical therapies, which ones would be most effective due to lack of clinical trials in this area. Thus, ocular GVHD is best managed in conjunction with ophthalmologist experienced in dealing with ocular GVHD.

In addition, special attention should be given to other risk factors for ocular complications, among which diabetes and hypertension are at the top. Thus, if a patient is on CNI or corticosteroids, then optimization of blood pressure and glycaemia control is imperative to reduce the risk of blindness due to retinopathy irrespective of the cause.

\subsubsection{Posterior Segment Complications}

Retinal and vitreous hemorrhages are not uncommon in HSCT patients and may happen with or without the presence of ocular GVHD (Yoo et al. 2017). This is complicated by the presence of thrombocytopenia early in transplant but also later in the course since both drugs and chronic GVHD can be associated with thrombocytopenia. Prompt referral to ophthalmologist is the key for preventing blindness; therefore, the practicing transplant clinician should have a high suspicion of retinopathy, retinal tears, or vitreous hemorrhages when a patient complains of "floaters" or just "decreased vision," which happens suddenly.

\subsubsection{Ocular Infections}

CMV infection is one the most widely studied ocular infections and can rapidly lead to retinitis, and since quite often IV antibiotics are required, prompt referral to ophthalmologist is mandatory. Apart from CMV, adenovirus is also a common virus and can lead to viremia if untreated. Moreover, unlike immunocompetent individuals, varicella zoster infection within hours or couple of days can lead to dissemination as well as postherpetic neuralgia, cranial nerve palsies, zoster paresis, meningoencephalitis, cerebellitis, myelopathy, and irreversible blindness.

Fungal infections in severely IS HSCT patients (particularly those on multiple IS for GVHD) can quickly lead to mortality; thus prompt referral for IV antifungals is indicated. Aspergillosis, mucormycosis, and candida have been reported in GVHD patients affecting the ocular tissues.

\subsubsection{Glaucoma}

Since the most common subtype of glaucoma (primary open-angle glaucoma) presents with gradual symptoms, its diagnosis is frequently missed in early phases. However, many risk factors in HSCT can predispose to glaucoma and can lead to blindness which include diabetes (allo-HSCT patients have four times higher risk of diabetes), retinopathy, and steroid use (for GVHD). Since the diagnosis of glaucoma is based in tonometry, gonioscopy, perimetry, and ophthalmoscopy, regular screening by the ophthalmologist is indicated.

\subsubsection{Cataract}

Cataract is the most common cause of blindness in the developed world. Risk factors in the HSCT patients include steroid use, total body irradiation, and diabetes. Since intraocular lens implantation (particularly via phacoemulsification) has become a widely performed procedure worldwide for the treatment of cataracts, early recognition and prompt treatment can help in preservation of vision. This procedure is associated with low rate of complications; however, prevention of cataracts by controlling the risk factors should be the management strategy in HSCT survivors (Table 48.1). 
Table 48.1 Ocular complications of HSCT

\begin{tabular}{|c|c|c|c|}
\hline & Risk factors/manifestations & Management & Survivorship issues \\
\hline $\begin{array}{l}\text { GVHD } \\
\text { associated }\end{array}$ & $\begin{array}{ll}\text { - } & \text { Dry eye syndrome } \\
& \text { (keratoconjunctivitis sicca)/ } \\
& \text { lacrimal gland dysfunction } \\
\text { - } & \text { Retinopathies } \\
\text { - } & \text { Cataracts } \\
\text { - } & \text { Infections }\end{array}$ & $\begin{array}{l}\text { - Topical: artificial tears, } \\
\text { steroids, CSA, scleral lenses, } \\
\text { autologous serum, surgical } \\
\text { procedures } \\
\text { - } \begin{array}{l}\text { Systemic: ECP, rituximab, } \\
\text { ibrutinib }\end{array}\end{array}$ & $\begin{array}{l}\text { Presence of ophthalmologist } \\
\text { in the long-term follow-up or } \\
\text { survivorship clinic }\end{array}$ \\
\hline $\begin{array}{l}\text { Non-GVHD } \\
\text { associated }\end{array}$ & $\begin{array}{ll}\text { - } & \text { Glaucoma } \\
\text { - } & \text { Cataract } \\
\text { - } & \text { Infection } \\
\text { - } & \text { Retinal/vitreous hemorrhage }\end{array}$ & $\begin{array}{ll}- & \text { Removal of risk factors } \\
- & \text { Surgery } \\
- & \text { Antimicrobials }\end{array}$ & \\
\hline
\end{tabular}

\section{Key Points}

- Ocular complications of HSCT are not restricted to GVHD, since both nonGVHD allo-HSCT and auto-HSCT recipients can suffer from cataracts, viral/fungal infections, glaucoma, and retinopathies.

- QoL compromise by ocular complications of HSCT is paramount due to ensuing blindness.

- Transplant centers should consider a close collaboration with ophthalmology teams both for treatment and preventative strategies. This could ideally be achieved in a multidisciplinary team in a long-term follow-up or a survivorship clinic.

\subsection{Oral Complications}

\subsubsection{Introduction}

Oral complications of HSCT can significantly affect the quality of the life (QoL) and can be a result of graft-versus-host-disease (GVHD) or can occur independent of it. Any oral complication, if severe, can lead to weight loss due to poor nutritional intake, mortality (especially due to cancer), aspiration pneumonia (especially in cases of oropharyngeal disorders), and psychologic sequelae (particularly disfigurement due to oral scleroderma, i.e., fish mouth) (Table 48.2).

\subsubsection{Oral GVHD}

Oral GVHD itself can lead to significant mortality and morbidity. GVHD-associated oral complications, e.g., mouth scleroderma, lichenification (resulting in lichenoid features and lichen planus), oral cancers, dental caries, salivary gland dysfunction (main manifestation being xerostomia), and mucoceles, are common complications of oral GVHD. In addition, avascular necrosis (AVN) of the mandible can occur as a complication of steroid therapy for GVHD (Treister et al. 2012).

The 2014 National Institutes of Health chronic GVHD consensus criteria places the scoring based on lichen-like features; however many of the associated patient-reported outcomes are not captured by this scoring system which can tremendously affect the QoL (Jagasia et al. 2015). Thus, involvement of specialists experienced in dealing with long-term HSCT complications is imperative for management of oral GVHD and its complications.

The management of oral GVHD requires both topical treatments and systemic therapy. Topical treatments may include local tacrolimus (i.e., protopic), PUVA, and topical steroids. Among systemic treatments for oral GVHD, extracorporeal photopheresis has one of the highest response rates, along with rituximab (Okamoto et al. 2006; Malik et al. 2014).

\subsubsection{Oral Cancers}

Carcinomas of the oral cavity are predominantly of squamous cell carcinoma type; however, other 
Table 48.2 Oral complications of hematopoietic stem cell transplantation

\begin{tabular}{|c|c|c|c|}
\hline & Risk factors & Management & Survivorship issues \\
\hline $\begin{array}{l}\text { GVHD } \\
\text { associated }\end{array}$ & $\begin{array}{l}\text { - Lichen planus } \\
\text { - } \text { Squamous cell carcinoma } \\
\text { - Aphthous ulcers } \\
\text { - } \quad \text { Fish mouth (scleroderma), carries, } \\
\quad \text { odynophagia, carries }\end{array}$ & $\begin{array}{l}\text { - } \quad \text { Topical: tacrolimus, } \\
\text { steroids, PUVA } \\
\text { - } \quad \text { Systemic: extracorporeal } \\
\text { photopheresis, rituximab, } \\
\text { ibrutinib }\end{array}$ & $\begin{array}{l}\text { - Presence of a dentist } \\
\text { and/or oral surgeon in } \\
\text { the long-term follow-up } \\
\text { or survivorship clinic }\end{array}$ \\
\hline $\begin{array}{l}\text { Non-GVHD } \\
\text { associated }\end{array}$ & $\begin{array}{l}\text { Osteonecrosis of the jaw, } \\
\text { dysgeusia, temporomandibular } \\
\text { joint disorders, mucositis, carries, } \\
\text { odynophagia }\end{array}$ & $\begin{array}{l}\text { Dental or oral surgery referral } \\
\text { for corrective action; surgery } \\
\text { in selected cases }\end{array}$ & \\
\hline
\end{tabular}

histologies can also manifest as oral cancers in HSCT survivors. The risks of developing oral cancers increase significantly post transplant to approximately sevenfold with the highest risk being in patients who were transplanted for bone marrow failure (BMF) syndromes especially dyskeratosis congenita and Fanconi's anemia (Rosenberg et al. 2003). Besides BMF syndromes, the most important risk factors for oral carcinomas include GVHD, radiation, and epigenetic factors (smoking). It is of utmost important that prevention strategies of oral cancers be discussed at each visit with special emphasis on smoking cessation (if applicable) and avoidance of other known risk factors, e.g., betel nut, tobacco chewing, or alcohol intake, since these are modifiable risks. Additionally, aggressive and early treatment of oral GVHD is essential to prevent this complication.

\subsubsection{Non-GVHD-Associated Oral Manifestations}

These include osteonecrosis of the jaw (ONJ), dysgeusia, temporomandibular disorders, gingivitis, and oral mucositis early in HSCT. Though certain symptoms like dysgeusia (Sato et al. 2017a, b) may seem trivial, they significantly affect the QoL, preservation of which is the sine qua non of managing late effects. Similarly, oral mucositis is one of the most challenging complications in early phases of HSCT with a high incidence in both reduced intensity and myeloablative regimens (Chaudhry et al. 2015). Similarly, ONJ can lead to multiple late effects of chronic pain and poor oral intake and can affect QoL tremendously (Hautmann et al. 2011). Thus, prompt referral to maxillofacial surgeon for management is imperative as soon as any symptoms start to develop.

\subsubsection{Needs Provision for Oral Surgery/Dentistry in HSCT}

Many experts recommend that dentistry and/or oral surgeons should be part of a multidisciplinary team managing late effects in both autologous and allogeneic HSCT survivors, and this concept was recently endorsed by the National Institutes of Health's Late Effects Initiative (Hashmi et al. 2017). Once the primary disease (for which HSCT was performed) is cured, it is essential that surveillance and preventative strategies be undertaken to alleviate the burden of comorbidities in these survivors; thus we agree that a long-term follow-up clinic should optimally have dentistry services available ad hoc if not on routine surveillance basis.

\section{Key Points}

- Oral GVHD and its associated complications can lead to significant compromise in QoL and can lead to cancers which can lead to fatal outcomes.

- Non-GVHD-associated complications also should be vigilantly dealt with as they tremendously affect QoL and include ONJ, dysgeusia, dental carries, gingivitis, and oral mucositis.

- Dentists and/or oral surgeons should ideally be a part of multidisciplinary teams of a long-term follow-up/survivorship clinic of HSCT survivors. 


\section{References}

Carpenter PA, Kitko CL, Elad S, et al. National institutes of health consensus development project on criteria for clinical trials in chronic graft-versus-host disease: V. The 2014 ancillary therapy and supportive care working group report. Biol Blood Marrow Transplant. 2015;21:1167-87.

Chaudhry HM, Bruce AJ, Wolf R, et al. The incidence and outcomes of oral mucositis among allogeneic stem cell transplantation patients: a systematic review and meta-analysis. Biol Blood Marrow Transplant. 2015;21:S245-6.

Hashmi SK, Bredeson C, Duarte RF, et al. National institutes of health blood and marrow transplant late effects initiative: the healthcare delivery working group report. Biol Blood Marrow Transplant. 2017;23:717-25.

Hautmann AH, Elad S, Lawitschka A, et al. Metabolic bone diseases in patients after allogeneic hematopoietic stem cell transplantation: report from the consensus conference on clinical practice in chronic graft-versushost disease. Transpl Int. 2011;24:867-79.

Jagasia MH, Greinix HT, Arora M, et al. National Institutes of Health consensus development project on criteria for clinical trials in chronic graft-versushost disease: I. The 2014 diagnosis and staging working group report. Biol Blood Marrow Transplant. 2015;21:389-401.

Malik MI, Litzow M, Hogan W, et al. Extracorporeal photopheresis for chronic graft-versus-host disease: a systematic review and meta-analysis. Blood Res. 2014;49:100-6.

Nassar A, Tabbara KF, Aljurf M. Ocular manifestations of graft-versus-host disease. Saudi J Ophthalmol. 2013;27:215-22.

Okamoto M, Okano A, Akamatsu S, et al. Rituximab is effective for steroid-refractory sclerodermatous chronic graft-versus-host disease. Leukemia. 2006;20:172-3.

Rosenberg PS, Greene MH, Alter BP. Cancer incidence in persons with Fanconi anemia. Blood. 2003;101: 822-6.

Sato T, Konuma T, Miwa Y, et al. A cross-sectional study on late taste disorders in survivors of allogeneic hematopoietic cell transplantation. Ann Hematol. 2017a;96:1841-7.

Sato T, Konuma T, Miwa Y, et al. A cross-sectional study on late taste disorders in survivors of allogeneic hematopoietic cell transplantation. Ann Hematol. 2017b;96:1841-7.

Townley JR, Dana R, Jacobs DS. Keratoconjunctivitis sicca manifestations in ocular graft versus host disease: pathogenesis, presentation, prevention, and treatment. Semin Ophthalmol. 2011;26:251-60.

Treister N, Duncan C, Cutler C, Lehmann L. How we treat oral chronic graft-versus-host disease. Blood. 2012;120:3407-18.

Yoo YS, Na KS, Shin JA, et al. Posterior eye segment complications related to allogeneic hematopoietic stem cell transplantation. Retina. 2017;37:135-43.

Open Access This chapter is licensed under the terms of the Creative Commons Attribution 4.0 International License (http://creativecommons.org/licenses/by/4.0/), which permits use, sharing, adaptation, distribution and reproduction in any medium or format, as long as you give appropriate credit to the original author(s) and the source, provide a link to the Creative Commons license and indicate if changes were made.

The images or other third party material in this chapter are included in the chapter's Creative Commons license, unless indicated otherwise in a credit line to the material. If material is not included in the chapter's Creative Commons license and your intended use is not permitted by statutory regulation or exceeds the permitted use, you will need to obtain permission directly from the copyright holder. 